\title{
Calidad de vida en pacientes con hepatitis C crónica en Colombia
}

\section{Quality of Life of Patients with Chronic Hepatitis C in Colombia}

Ismael de Jesús Yepes Barreto, PhD, ${ }^{1}$ Zenen Carmona Meza, ${ }^{1}$ María Nicol Múnera Contreras, MD. ${ }^{2}$

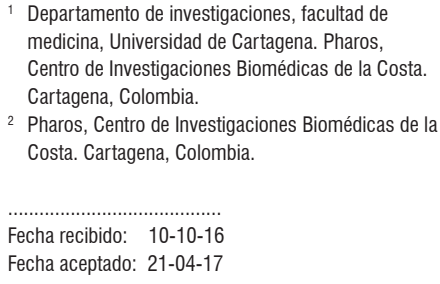
medicina, Universidad de Cartagena. Pharos, Centro de Investigaciones Biomédicas de la Costa. Cartagena, Colombia.

2 Pharos, Centro de Investigaciones Biomédicas de la Costa. Cartagena, Colombia.

Fecha recibido: $10-10-16$

Fecha aceptado: $21-04-17$

\begin{abstract}
Resumen
Introducción: los datos sobre calidad de vida en pacientes con hepatitis $\mathrm{C}$ son escasos en nuestro medio. A la fecha, no hay estudios que hayan determinado la carga de la enfermedad en esta población en el territorio colombiano. Objetivos: determinar la calidad de vida en pacientes con hepatitis $C$ crónica e identificar sus factores determinantes utilizando la encuesta SF-36 V2. Metodología: estudio transversal analítico realizado desde el 1 de junio de 2015 hasta el 31 de agosto de 2015. Un encuestador entrenado aplicó la encuesta SF36 V2 en su versión en castellano (Qualitimetric Inc.). La regresión lineal multivariante identificó los factores relacionados con la calidad de vida relacionada con la salud (CVRS). El índice de Charlson estimó el peso de las comorbilidades de la muestra. Resultados: 41 pacientes estuvieron disponibles para el análisis. La media de edad fue de 57 años (desviación estándar [DE]: 12,6). El 50\% de los sujetos presentaban cirrosis/ fibrosis avanzada. El Resumen del componente físico (RCF) y el Resumen del componente mental (RCM) fueron de 48,2 y 45,6 respectivamente. El análisis de regresión lineal univariante identificó al sexo, la edad, el índice de Charlson y la presencia de cirrosis hepática como los principales determinantes de la calidad de vida. El índice de Charlson expresado como la probabilidad de supervivencia a 10 años fue el único predictor independiente del SF-6D. Conclusión: la edad, el sexo y la cirrosis hepática se identificaron como determinantes de la CVRS. El índice de Charlson fue el único predictor independiente de SF-6D. La implementación de programas multidisciplinarios es necesaria para el tratamiento integral de los pacientes infectados.
\end{abstract}

\section{Palabras clave}

Hepatitis C, Colombia, calidad de vida, cirrosis hepática, América Latina, hepatitis.

\begin{abstract}
Introduction: Data on quality of life in patients with hepatitis $C$ are scarce in our country. To date, no study has determined the burden of chronic hepatitis $C$ in the population of Colombia. Objectives: The objective of this study was to determine the quality of life in patients with chronic hepatitis $C$ and to identify its determinants using the SF36V2 survey. Methodology: This cross-sectional analytical study was conducted between June 1,2015 and August 31, 2015. A trained interviewer used the Spanish version of the SF-36 V2 survey (Quality Metric Inc.). Then multivariate linear regression identified factors related to Health Related Quality of Life (HRQL). The Charlson Comorbidity Index was used to estimate the weights of comorbidities found in the sample. Results: Forty-one patients were available for analysis. The mean age was 57 years (SD 12.6). Fifty percent of the subjects had advanced cirrhosis and/or fibrosis. The physical component summary (PCS) and mental component summary (MCS) were 48.2 and 45.6, respectively. Univariate linear regression analysis identified sex, age, Charlson Comorbidity Index and presence of liver cirrhosis as the main determinants of quality of life. The Charlson Comorbidity Index 10 -year survival probability was the only independent predictor of SFD6. Conclusion: Age, sex and hepatic cirrhosis were identified as determinants of HRQLL. The Charlson index was the only independent predictor of SFD6. The implementation of multidisciplinary programs is necessary for comprehensive treatment of infected patients.
\end{abstract}

\section{Keywords}

Hepatitis C, Colombia, quality of life, hepatic cirrhosis, Latin America, hepatitis. 


\section{INTRODUCCIÓN}

Se estima que aproximadamente 6,8-8,9 millones de personas están infectadas por el virus de la hepatitis $\mathrm{C}(\mathrm{VHC})$ en Latinoamérica, de los que $<1 \%$ llegan a recibir tratamiento antiviral (1). La prevalencia en Colombia se desconoce; sin embargo, la Organización Panamericana de la Salud (OPS) ha reportado que entre el $0,7 \%$ y el $0,9 \%$ de los donantes presentan anticuerpos frente al VHC $(2,3)$. La trasmisión nosocomial es la fuente más frecuente de infección y se ha identificado la transfusión sanguínea antes de 1994 como el principal factor de riesgo para contraer el virus en Colombia y otros países latinoamericanos $(4,5)$.

Aproximadamente, entre 250000 y 350000 personas se encuentran crónicamente infectadas por el VHC en el país, de las cuales fallecen anualmente entre 2150 y 2400 a causa de cirrosis hepática y cáncer de hígado, ocasionando entre 84,8 y 106,1 años ajustados por discapacidad (DALY) por cada 100000 personas durante el 2014 (6).

Sin embargo, el impacto de la infección por el VHC no se limita solamente a la aparición de cirrosis y cáncer hepatocelular; de hecho, se ha observado un detrimento de la calidad de vida relacionada con la salud (CVRS) independientemente del estadio de la enfermedad hepática (7).

Aunque algunos estudios realizados en Brasil y Estados Unidos han estudiado ampliamente la CVRS en esta población, las diferencias socioculturales no permiten extrapolar estos resultados a nivel local (8-12).

A la fecha, en Colombia no se ha realizado ningún estudio que haya determinado la carga de la enfermedad en esta población, lo que impide conocer su impacto real en el medio y dificultando la interpretación de estudios de costoefectividad que faciliten la toma de decisiones clínicas ante el advenimiento de los nuevos antivirales al país.

El objetivo de este trabajo es describir la CVRS en una población de pacientes infectados por el VHC en Colombia e identificar sus factores determinantes.

\section{METODOLOGÍA}

Estudio transversal analítico realizado desde el 1 de junio de 2015 hasta el 31 de agosto de 2015. Se incluyeron de forma sistemática a todos los pacientes con diagnóstico de hepatitis $\mathrm{C}$ crónica por ensayo por inmunoadsorción ligado a enzimas (ELISA) y con una carga viral confirmatoria. Un encuestador entrenado aplicó la encuesta SF-36 V2 en su versión en español a cada uno de los pacientes y se recolectó información epidemiológica, clínica y analítica actualizada a la fecha de inclusión en el estudio. Los pacientes con encefalopatía hepática o con limitaciones físicas o mentales para diligenciar la SF-36 V2 fueron excluidos. Los pacientes con ascitis no se excluyeron para que la muestra fuera representativa de pacientes en todos los estadios de la enfermedad. Qualitimetric Inc. otorgó el permiso para el uso de la encuesta SF-36 V2 y certificó que los investigadores del International Quality of Life Assessment Project Approach (IQOLA) (13) prepararon una versión en español de la encuesta que fue revisada por lingüistas e investigadores colombianos quienes confirmaron que esta traducción era aceptable en Colombia. Todos los pacientes firmaron un consentimiento informado antes de su inclusión en el estudio. Este protocolo contó con la aprobación del comité de ética del Centro Cardiovascular Santa Lucía de la ciudad de Cartagena.

\section{Elastografía transitoria}

Para la estimación de la rigidez hepática se utilizó la elastografía transitoria mediante un fibroscan (Ecosense) serie 502. Este aparato emite una onda vibratoria que atraviesa el parénquima hepático, la velocidad a la cual recorre esta onda el órgano genera una estimación del grado de fibrosis que se expresa en kilopascales $(\mathrm{kPa})$; a mayor velocidad mayor grado de fibrosis y viceversa. Todos los pacientes guardaron ayuno durante al menos 8 horas. El procedimiento se efectuó en decúbito supino con el brazo derecho en abducción máxima, las determinaciones se realizaron sobre la línea axilar media. Para la evaluación de la rigidez hepática se tomó en cuenta el valor mediano de 10 estimaciones, los resultados que incumplían los criterios de calidad del fabricante no se tomaron en cuenta para el análisis. Un rango intercuartílico $<30 \%$ y un porcentaje de validez (número de mediciones válidas/mediciones totales) $>60 \%$ se consideraron indispensables para evaluar la fiabilidad del resultado. El punto de corte utilizado para el diagnóstico de cirrosis fue de $14,5 \mathrm{kPa}$.

\section{SF-36 V2}

La SF-36 V2 consiste en un cuestionario de 36 ítems que evalúan 8 áreas de la CVRS y genera, además, 2 indicadores que resumen los componentes físico y mental de la escala, así:

- Funcionamiento físico (FF): evalúa la limitación de las actividades físicas por la enfermedad.

- Papel físico (PF): evalúa el impacto de la salud física sobre el trabajo y las actividades de la vida diaria.

- Dolor corporal (DC): evalúa las limitaciones del paciente a causa del dolor.

- Salud general (SG): evalúa cómo el sujeto ve su salud personal y su riesgo de empeoramiento.

- Vitalidad (VT): evalúa el grado de cansancio o de energía de un individuo.

- Funcionamiento social (FS): estima cuánto interfieren los problemas físicos o emocionales con las actividades sociales normales. 
- Papel emocional (PE): determina el impacto de los problemas emocionales en el trabajo y las actividades de la vida diaria.

- Salud mental (SM): evalúa el estado de ánimo general (deprimido, feliz, entre otros).

- RCF: resume el componente físico de la SF-36 V2: FF, PF, DC y SG.

- RCM: resume el componente mental de la FS-36 V2: VT, FS, PE y SM.

Cada una de las escalas de la SF-36 V2 tiene un rango de 0 a 100 , en la que 100 es el mejor puntaje posible. Diferencias en el RCF y RCM $>3$ puntos son consideradas relevantes.

El SF-6D es un índice (utilidades) en salud derivado de la SF-36 desarrollado en el Reino Unido, que se calcula otorgándole determinado peso a diferentes aspectos de la encuesta y que facilita las evaluaciones económicas en salud. Sus valores fluctúan entre 0 y 1 , representando el peor y el estado de salud ideal, respectivamente. Diferencias de 0,03 puntos se consideran clínicamente significativas.

\section{Índice de comorbilidad de Charlson}

El índice de comorbilidad de Charlson predice la mortalidad para un paciente teniendo en cuenta 22 condiciones. A cada condición se le asigna una puntuación de 1, 2, 3 o 6 dependiendo del riesgo de fallecer asociado con esta condición y se calcula la probabilidad de supervivencia del paciente. La hepatitis crónica y la cirrosis hepática se consideraron como enfermedad hepática leve y grave, respectivamente.

\section{Análisis estadístico}

Las variables numéricas se expresaron como la media \pm desviación estándar (DE) y las categóricas en forma de porcentaje, respectivamente. La $t$-student y la ji cuadrada se utilizaron para la comparación entre grupos según correspondiera. Para identificar los factores determinantes de la calidad de vida se realizó un análisis de regresión lineal univariante y multivariante en el que la variable dependiente fue el índice SF-6D, el RCM y el RCF. Para facilitar el análisis del índice de Charlson se consideró una variable continua que expresaba la probabilidad de supervivencia durante los siguientes 10 años. La relación se consideró estadísticamente significativa con un valor de $\mathrm{p}<0,05$.

\section{RESULTADOS}

Finalmente, 41 pacientes estuvieron disponibles para el análisis. La muestra estuvo constituida por un número similar de hombres y mujeres. La media de edad fue de 57 años (DE: 12,6). El genotipo más frecuente fue el $1 \mathrm{~b}$ y aproximadamente un 50\% de los sujetos presentaban cirrosis/fibrosis avanzada (F3-F4). La probabilidad promedio de supervivencia a los 10 años en función al índice de Charlson fue de 53\% de forma global (DE: 36). 20 pacientes habían recibido tratamiento antiviral, de los cuales 10 alcanzaron respuesta virológica sostenida. Las características basales de la muestra pueden observarse en la Tabla 1.

Tabla 1. Características clínicas y demográficas basales

\begin{tabular}{|c|c|}
\hline$n$ & 41 \\
\hline Sexo masculino & $22(53,7)$ \\
\hline Edad & $57(12,6)$ \\
\hline \multicolumn{2}{|l|}{ Régimen de salud } \\
\hline Medicina prepagada & $9(22)$ \\
\hline Contributivo & $27(66)$ \\
\hline Subsidiado & $5(12)$ \\
\hline Diabetes & $5(9,3)$ \\
\hline Hipertensión & $16(30)$ \\
\hline Dislipidemia & $6(11)$ \\
\hline Obesidad & $3(5,6)$ \\
\hline \multicolumn{2}{|l|}{ Genotipo } \\
\hline $1 a$ & $1(2,4)$ \\
\hline $1 \mathrm{~b}$ & $13(32)$ \\
\hline 2 & $2(5)$ \\
\hline 4 & $1(2,4)$ \\
\hline Desconocido & $21(51)$ \\
\hline Fibroscan & $13,6(14,4)$ \\
\hline Tratamiento antiviral & $20(49)$ \\
\hline RVS & $10(50)$ \\
\hline \multicolumn{2}{|l|}{ Fibrosis } \\
\hline Desconocido & $3(7)$ \\
\hline F0 & $13(32)$ \\
\hline F1 & $2(5)$ \\
\hline F2 & $2(5)$ \\
\hline F3 & $5(12,2)$ \\
\hline Cirrosis & $16(39)$ \\
\hline \multicolumn{2}{|l|}{ Child-Pugh-Turcotte } \\
\hline$A$ & $11(68)$ \\
\hline$B$ & $5(32)$ \\
\hline INR & $1,18(0,27)$ \\
\hline Albúmina & $3,8(0,6)$ \\
\hline Bilirrubina & $0,96(0,6)$ \\
\hline Creatinina & $0,9(0,26)$ \\
\hline Várices & $9(56,3)$ \\
\hline Ascitis & $3(18,8)$ \\
\hline Encefalopatía & $2(12,5)$ \\
\hline
\end{tabular}

INR: índice internacional normalizado; RVS: resistencia vascular sistémica. 
El RCF y el RCM fueron de 48,2 y 45,6 respectivamente. Estos valores son inferiores a los que se reportan para poblaciones sin enfermedades crónicas y son similares a los descritos en pacientes con diabetes y anemia. Las subescalas con puntuaciones más bajas fueron la VT, SG, DC, SM y PF (Tabla 2).

Tabla 2. Escalas y subescalas de la encuesta SF-36 V2

\begin{tabular}{lcccc}
\hline & Media & Mediana & P25 & P75 \\
\hline FF & $72(23,3)$ & 75 & 55 & 90 \\
PF & $66,4(33,3)$ & 75 & 37,5 & 100 \\
DC & $65,1(26,5)$ & 62 & 41 & 92 \\
SG & $62,9(19,8)$ & 65 & 52 & 77 \\
VT & $58,5(22,6)$ & 62,5 & 43,7 & 71,8 \\
FS & $71,3(28,9)$ & 75 & 50 & 100 \\
PE & $68(27,3)$ & 66,6 & 50 & 91,6 \\
SM & $66(21,5)$ & 70 & 50 & 85 \\
RCF & $48,2(9,1)$ & 49,4 & 40,3 & 55 \\
RCM & $45,6(11,1)$ & 46,6 & 36,3 & 55,4 \\
SF-6D & $0,70(0,15)$ & 0,7 & 0,57 & 0,84 \\
\hline
\end{tabular}

\section{Factores asociados con la CVRS}

Un análisis bivariado inicial determinó que el sexo femenino se asoció con una disminución significativa en las escalas de DC, PE, SM, RCM y SF-6D. De igual manera, la presencia de cirrosis hepática tuvo una asociación negativa en 8 de las 10 escalas y subescalas de la SF-36 V2 y del SF-6D. Aunque los sujetos que no alcanzaron la respuesta virológica sostenida $(\mathrm{n}=31)$ después del tratamiento tuvie- ron puntuaciones inferiores en todas las escalas, solamente alcanzaron diferencias estadísticamente significativas en las escalas VT y FF (Tabla 3). De manera similar, todas las escalas y el SF-6D disminuyeron a medida que se incrementaban el grado de fibrosis hepática estimada por elastografía transitoria; encontrándose la asociación más estrecha con la FF, PF, SG, VT, PE y RCF (Tabla 4); sin embargo, esta relación desapareció al excluir a los pacientes con cirrosis.

$\mathrm{El}$ análisis de regresión lineal univariante identificó el sexo, edad, índice de Charlson y presencia de cirrosis hepática como los principales determinantes del SF-6D (Tabla 5). El índice de Charlson también presentó una asociación lineal positiva con el RCM y RCF. Mientras que la edad y los valores elastográficos presentaron una asociación lineal negativa con el RCF. Los pacientes de género masculino tuvieron puntuaciones más elevadas en el RCM que sus contrapartes femeninas (Tabla 6 y 7 ).

El índice de Charlson, expresado como la probabilidad de supervivencia a 10 años, fue el único predictor independiente del índice de utilidades en este estudio (Tabla 8).

\section{DISCUSIÓN}

Este estudio aporta los primeros datos sobre calidad de vida y utilidades en salud en pacientes con hepatitis $\mathrm{C}$ crónica en Colombia. Esta información puede ser útil en la implementación de programas integrales en el tratamiento de la enfermedad y en la realización de estudios de costo-efectividad para los nuevos antivirales disponibles en el país.

El RCM y RCF promedio en sujetos sin enfermedades crónicas en los Estados Unidos es de 52,2 y 54,3 respectivamente. En este estudio el promedio para cada una de

Tabla 3. Relación entre el sexo, la presencia de cirrosis y de repuesta virológica sostenida con las diferentes escalas y subescalas del SF-36 V2

\begin{tabular}{lccccccccc}
\hline & $\begin{array}{c}\text { Femenino frente a } \\
\text { masculino }\end{array}$ & IC 95\% & $\mathbf{p}$ & $\begin{array}{c}\text { No cirrosis frente } \\
\text { a cirrosis }\end{array}$ & IC 95\% & $\mathbf{p}$ & $\begin{array}{c}\text { No RVS } \\
\text { frente a RVS }\end{array}$ & IC 95\% & $\mathbf{p}$ \\
\hline FF & $-6,8$ & $(-21,6)-8,0$ & 0,35 & 16,6 & $1,7-31,5$ & 0,03 & $-23,5$ & $(-47,1)-0,15$ & 0,05 \\
PF & $-17,8$ & $(-38,4)-2,8$ & 0,08 & 23,9 & $2,4-45,3$ & 0,03 & $-13,5$ & $(-46,7)-19,4$ & 0,39 \\
DC & -19 & $(-35,1)-(-3,4)$ & 0,01 & 8,8 & $(-8,8)-26,6$ & 0,31 & $-6,5$ & $(-29,5)-16,4$ & 0,55 \\
SG & $-4,5$ & $(-17,2)-8,13$ & 0,47 & 20,3 & $8,2-32,4$ & 0,002 & $-4,5$ & $(-21,6)-12,4$ & 0,57 \\
VT & $-11,6$ & $(-25,6)-2,3$ & 0,1 & 19 & $5,0-33,0$ & 0,009 & $-19,3$ & $(-38,8)-0,14$ & 0,05 \\
FS & $-12,7$ & $(-30.9)-5,3$ & 0,16 & 19,6 & $0,88-38,3$ & 0,04 & $-22,5$ & $(-52,9)-7,9$ & 0,13 \\
PE & $-17,3$ & $(-33,9)-(-0,7)$ & 0,04 & 24,7 & $7,8-41,7$ & 0,005 & $-0,2$ & $(-29,5)-29,1$ & 0,98 \\
SM & $-19,2$ & $(-31,5)-(-6,8)$ & 0,003 & 20,5 & $7,6-33,4$ & 0,003 & $-7,3$ & $(-28,6)-13,9$ & 0,47 \\
RCF & $-2,8$ & $(-8,6)-2,8$ & 0,32 & 5,1 & $(-0,94)-11,15$ & 0,09 & $-6,2$ & $(-15,5)-(-3,0)$ & 0,17 \\
RCM & $-8,5$ & $(-15,1)-(-1,9)$ & 0,01 & 10,9 & $4,2-17,6$ & 0,002 & $-3,5$ & $(-14,9)-7,9$ & 0,52 \\
SF-6D & $-0,1$ & $(-0,20)-(-0,013)$ & 0,02 & 0,11 & $0,017-0,21$ & 0,02 & $-0,09$ & $(-0,23)-0,04$ & 0,18 \\
\hline
\end{tabular}


Tabla 4. Relación entre la elastografía transitoria y las diferentes escalas y subescalas del SF-36 V2

\begin{tabular}{lcc}
\hline & Fibroscan & $\mathbf{p}$ \\
\hline FF & $-0,5$ & 0,004 \\
PF & $-0,38$ & 0,03 \\
DC & $-0,28$ & 0,11 \\
SG & $-0,34$ & 0,05 \\
VT & $-0,4$ & 0,02 \\
FS & $-0,32$ & 0,07 \\
PE & $-0,44$ & 0,01 \\
SM & $-0,24$ & 0,17 \\
RCF & $-0,4$ & 0,02 \\
RCM & $-0,33$ & 0,06 \\
SF-6D & $-0,32$ & 0,07 \\
\hline
\end{tabular}

Tabla 6. Regresión lineal univariante. Variable dependiente RCF (resumen del componente físico)

\begin{tabular}{llll}
\hline & \multicolumn{1}{c}{$\mathbf{b}$} & \multicolumn{1}{c}{ IC 95\% } & \multicolumn{1}{c}{$\mathbf{p}$} \\
\hline Sexo & 0,15 & $(-2,8)-8,6$ & 0,32 \\
Cirrosis & 0,25 & $(-11,1)-0,94$ & 0,09 \\
RVS & 0,028 & $(-6,9)-8,0$ & 0,87 \\
Supervivencia & 0,47 & $0,04-0,19$ & 0,003 \\
Edad & $-0,39$ & $(-0,47)-(-0,07)$ & 0,01 \\
Fibroscan & $-0,4$ & $(-0,5)-(-0,03)$ & 0,02 \\
\hline
\end{tabular}

Tabla 8. Regresión lineal multivariante. Variable dependiente SF-6D

\begin{tabular}{llll}
\hline & \multicolumn{1}{c}{ b } & \multicolumn{1}{c}{ IC 95\% } & \multicolumn{1}{c}{$\mathbf{p}$} \\
\hline Sexo & 0,14 & $(-0,05)-0,15$ & 0,37 \\
Cirrosis & 0,2 & $(-0,08)-0,21$ & 0,38 \\
Índice de Charlson & 0,69 & $0,000-0,006$ & 0,02 \\
Edad & 0,04 & $(-0,05)-0,006$ & 0,84 \\
\hline
\end{tabular}

ellas fue de 48,2 y 45,6 respectivamente; estos valores son similares a los encontrados en otras enfermedades crónicas como la diabetes y la depresión (14).

Las subescalas que valoraban la VT, la SG, el DC, la SM y el impacto de la salud física sobre el trabajo o las actividades de la vida diaria fueron las áreas más afectadas en esta cohorte de pacientes.

Una revisión reciente (15) de los estudios cualitativos más importantes publicados sobre calidad de vida en la población con hepatitis C crónica en Reino Unido, Estados Unidos y Australia para identificar los aspectos que afectaban la calidad de vida de estos sujetos, señaló la fatiga, la debilidad y el cansancio como los síntomas físicos más frecuentes, y fueron la causa principal de la disminución de la actividad física y deportiva $(16,17)$. Además, la culpa
Tabla 5. Regresión lineal univariante. Variable dependiente SF-6D

\begin{tabular}{llll}
\hline & \multicolumn{1}{c}{$\mathbf{b}$} & \multicolumn{1}{c}{ IC 95\% } & \multicolumn{1}{c}{$\mathbf{p}$} \\
\hline Sexo & 0,34 & $0,01-0,20$ & 0,02 \\
Cirrosis & $-0,36$ & $(-0,21)-(-0,17)$ & 0,02 \\
RVS & $-0,04$ & $(-0,14)-0,11$ & 0,79 \\
Índice de Charlson & 0,56 & $0,001-0,004$ & $<0,0001$ \\
Edad & $(-0,38)$ & $(-0,008)-(-0,001)$ & 0,015 \\
Fibroscan & $-0,32$ & $(-0,008)-0,00$ & 0,07 \\
\hline
\end{tabular}

Tabla 7. Regresión lineal univariante. Variable dependiente RCM (resumen del componente mental)

\begin{tabular}{llll}
\hline & \multicolumn{1}{c}{$\mathbf{b}$} & \multicolumn{1}{c}{ IC 95\% } & \multicolumn{1}{c}{$\mathbf{p}$} \\
\hline Sexo & 0,38 & $1,9-15,1$ & 0,01 \\
Cirrosis & $-0,01$ & $(-3,1)-2,9$ & 0,95 \\
RVS & $-0,11$ & $(-10,5)-5,7$ & 0,55 \\
Supervivencia & 0,55 & $0,08-0,25$ & $<0,001$ \\
Edad & $-0,24$ & $(-0,46)-0,06$ & 0,13 \\
Fibroscan & $-0,33$ & $(-0,49)-0,017$ & 0,06 \\
\hline
\end{tabular}

por considerarse una carga económica para la familia y por conductas adictivas previas $(18,19)$, el estigma social de la infección $(16,19)$ y la posibilidad de ser una fuente de contagio para otros son un motivo frecuente de preocupación para estos pacientes. Estos trabajos también señalaron que la ansiedad, el miedo, la rabia y la depresión son frecuentes entre los infectados $(16,17,19,20)$. También se hicieron patentes algunos rasgos de comportamiento negativos. Los más comunes fueron mantener en secreto la enfermedad ante familiares y amigos, actitudes agresivas, la incertidumbre sobre la esperanza de vida, su salud en general y el estado financiero, actitud social desagradable y comportamientos irracionales con la pareja $(19,20)$.

Varios de estos estudios también reportaron una disminución de la actividad social y laboral por fatiga o por el estrés emocional asociado con la infección. De igual manera, se encontró una disminución de la actividad sexual por el miedo a la trasmisión de la infección o por pérdida de la libido $(16,21)$.

Esta investigación tuvo un enfoque predominantemente cuantitativo utilizando una escala validada para la medición de la CVRS y generación de índices de utilidades, por lo que no se recogió información cualitativa sobre las 
emociones y preocupaciones específicas de los pacientes. Sin embargo, las subescalas (VT, SG, PF, SM y DC) con menores puntuaciones en este estudio pueden asociarse de forma intuitiva con las preocupaciones de los pacientes reportadas en otros países (fatiga, ansiedad, cansancio, rabia, miedo, culpa, entre otras). En cualquier caso, es importante desarrollar investigaciones cualitativas que nos permitan profundizar en las preocupaciones, síntomas físicos, las reacciones psicológicas y sociales específicas de nuestros pacientes para la implementación de programa de atención integrales que nos permitan disminuir de forma eficaz la carga de la enfermedad.

El sexo femenino se asoció con una disminución significativa de la CVRS. El SF-6D, RCF y el RCM disminuyeron significativamente en este grupo con diferencias de $-0,10$ puntos $(\mathrm{p}=0,02),-8,5(\mathrm{p}=0,01)$ y $-2,8(\mathrm{p}=0,32)$, respectivamente. Un estudio reciente publicado en Colombia que estimó la calidad de vida en 44 pacientes con cirrosis (5), entre los cuales 15 presentaban una etiología viral, también identificó al sexo femenino como un determinante de la calidad de vida en esa población. Estos estudios junto a otros realizados en Norteamérica (8) señalan el género como uno de los principales determinantes de la calidad de vida en pacientes con enfermedad hepática crónica. Esta circunstancia debe tenerse en cuenta en el diseño de las guías de atención y de las políticas nacionales para el control y tratamiento de la enfermedad.

La edad se asoció con una disminución del SF-6D $(\mathrm{p}=0,015)$, lo cual es previsible teniendo en cuenta que las complicaciones de la hepatitis $\mathrm{C}$ y de otras enfermedades crónicas prevalentes se incrementa a medida que la población envejece. Otros estudios realizados en Latinoamérica también han observado hallazgos similares (9).

Los sujetos que alcanzaron RVS después del tratamiento tuvieron un incremento en todas las escalas, subescalas del SF-36V2 y en el SF-6D. Sin embargo, no se observó ninguna asociación significativa con el RCM, el RCF o el SF-6D en el análisis multivariante. Es probable que el escaso número de sujetos que la alcanzaron no haya permitido encontrar la asociación positiva entre el control de la replicación viral y la CVRS que se ha observado habitualmente con el control de la infección $(22,23)$.

Por otra parte, la presencia de cirrosis se asoció con valores más bajos en 8 de las 10 diferentes escalas y subescalas del SF-36 V2 y del índice de utilidad de forma significativa. Esta relación parece independiente de la presencia de las complicaciones de la enfermedad teniendo en cuenta que la mayor parte de los pacientes con cirrosis de la muestra se encontraba en la fase compensada de la enfermedad y solamente 3 habían presentado ascitis y 2 de ellos encefalopatía.

La relación lineal observada entre la elastografía transitoria y diferentes subescalas de la SF-36V2 sugiere que la calidad de vida disminuye a medida que se incrementa el grado de fibrosis hepática en los pacientes infectados, pero esta relación desapareció completamente cuando se excluyeron a los pacientes con cirrosis, lo que señala nuevamente que la aparición de la cirrosis es el punto de inflexión para el inicio de una etapa más agresiva de la enfermedad y un detrimento significativo de la CVRS.

El índice de Charlson es una escala que permite calcular la supervivencia de un paciente basándose en la presencia de 22 condiciones y es un indicador del peso de las comorbilidades dentro de una población determinada. La esperanza de vida de los pacientes a 10 años calculada por el índice de Charlson fue del 53\%; aunque significativamente baja, no es sorprendente porque al tratarse de una población infectada por el VHC se consideró que todos los pacientes presentaban una enfermedad hepática leve, y moderada o grave si presentaban una cirrosis establecida. Este hecho junto a la imposibilidad de introducir el impacto de la respuesta virológica sostenida dentro de los cálculos del índice, claramente influyó de forma negativa sobre la supervivencia calculada de la muestra.

Un estudio que incluyó 4781 pacientes infectados por hepatitis $\mathrm{C}$ en los Estados Unidos (8) determinó los factores asociados con la aparición de depresión y con el deterioro de la salud física de estos pacientes. Al igual que en nuestro estudio, el sexo femenino y las comorbilidades estimadas a través del índice de Charlson se asociaron con un detrimento del estado físico y mental de los pacientes. Algunos otros factores como el estrés y la situación laboral también tuvieron un efecto negativo sobre la CVRS. Desafortunadamente, no pudieron ser analizados en nuestro estudio por la carencia de herramientas válidas en nuestro medio para cuantificar el estrés y por el escaso número de sujetos que se encontraban desempleados.

El índice de Charlson fue el único predictor independiente del SF-6D, el RCF y el RCM en un modelo de regresión lineal multivariante que sugiere que los determinantes del bienestar de estos pacientes no dependen exclusivamente de la enfermedad hepática sino también del adecuado tratamiento de las comorbilidades asociadas mediante el apoyo de la implementación de programas integrales y multidisciplinarios para la atención de los pacientes infectados por el VHC en nuestro país.

El tamaño de la muestra fue, sin duda, la limitación más importante del estudio, lo que dificultó la generalización de estos resultados. Sin embargo, el difícil diagnóstico de la infección en el país ha evitado la obtención de muestras de mayor tamaño, es posible que sea necesario un esfuerzo conjunto de los diferentes actores que participan en el proceso de atención de esta población (entes gubernamentales, sociedades científicas, asociaciones de pacientes, instituciones prestadoras de servicios con programas de hepatología 
e infectología, entre otros) para fomentar la investigación en esta y otras áreas relevantes de la enfermedad en muestras de mayor tamaño que permitan obtener resultados más sólidos.

La alta proporción de pacientes en estadio F3-F4 de la muestra sugiere un posible sesgo de selección similar al que se observa en instituciones de alta complejidad adonde son remitidos los pacientes de mayor gravedad. Es importante anotar que esta muestra se obtuvo de la consulta externa de gastroenterología y hepatología de un centro de primer nivel de atención a donde son remitidos la mayor parte de los pacientes con hepatitis $\mathrm{C}$ de 4 de las más grandes empresas promotoras de servicios de salud (EPS) de la ciudad de Cartagena y, aunque es factible que un patrón sistemático de remisión a nuestra institución haya provocado un sesgo de selección, también debe considerarse la posibilidad de que, en efecto, una gran proporción de los pacientes infectados en la población general se encuentren en estadios avanzados porque la ausencia de programas efectivos de cribado, el carácter silente de la enfermedad y las barreras de acceso al tratamiento hayan permitido la progresión hasta estas fases. De hecho, teniendo en cuenta que el pico más alto de exposición al virus en nuestro país fue hace más de 20 años a través de la trasfusión de hemoderivados antes de 1994 y conociendo la historia natural de la infección podríamos especular que el número de pacientes con cirrosis hepática secundaria a la hepatitis $\mathrm{C}$ en Colombia debe incrementarse durante los próximos años.

En todo caso, a pesar de estas limitaciones, estos datos son los primeros reportados sobre la CVRS en pacientes infectados con hepatitis $\mathrm{C}$ en Colombia y aportan información relevante sobre una enfermedad de la que se sabe aún muy poco en el país.

\section{CONCLUSIÓN}

La edad, el sexo y la cirrosis hepática se identificaron como los determinantes de la CVRS. El índice de Charlson fue el único predictor independiente de SF-6D. La implementación de programas multidisciplinarios son necesarios para el tratamiento integral de los pacientes infectados.

\section{Agradecimientos}

A todos los pacientes que de forma desinteresada participaron en este estudio y a Qualitimetric Inc. por haber facilitado la encuesta en su versión en español de forma gratuita.

\section{Declaración de conflicto de interés}

Los autores no tienen conflicto de intereses que declarar con respecto a la presentación de resultados de esta investigación.

\section{Financiación}

Esta investigación no tuvo ninguna financiación externa y se llevó a cabo con recursos propios de la Universidad de Cartagena.

\section{REFERENCIAS}

1. Kershenobich D, Razavi HA, Sanchez-Avila JF, et al. Trends and projections of hepatitis $C$ virus epidemiology in Latin America. Liver Int. 2011;31 Suppl 2:18-29. Doi: https:// doi.org/10.1111/j.1478-3231.2011.02538.x

2. Gabriel A. Schmunis FZ, Francisco Pinheiro, et al. Risk for transfusion-transmitted infectious diseases in Central and South America. Emerg Infect Dis. 1998;4(1):5-11.

3. Schmunis GA, Zicker F, Cruz JR, et al. Safety of blood supply for infectious diseases in Latin American countries, 1994-1997. Am J Trop Med Hyg. 2001;65(6):924-30. Doi: https://doi.org/10.4269/ajtmh.2001.65.924

4. Botero R. Los nuevos tratamientos de hepatitis C: Perspectivas latinoamericanas. Clinical Liver Disease. 2015;5(1):11-13.

5. Yepes IJ LB, Caez C, y cols. Factores de riesgo para la infección por el virus de la hepatitis $\mathrm{C}$ en la Costa Caribe colombiana: un estudio de casos y controles. Biomédica. 2016;36(4):564-71.

6. Alvis N. Burden of disease of hepatitis C in Colombia. Value in Health. 2015;18(3):A250-1.

7. Bonkovsky HL, Snow KK, Malet PF, et al. Health-related quality of life in patients with chronic hepatitis $\mathrm{C}$ and advanced fibrosis. J Hepatol. 2007;46(3):420-31. Doi: https:// doi.org/10.1016/j.jhep.2006.10.009

8. Boscarino JA, Lu M, Moorman AC, et al. Predictors of poor mental and physical health status among patients with chronic hepatitis $\mathrm{C}$ infection: the Chronic Hepatitis Cohort Study (CHeCS). Hepatology. 2015;61(3):802-11. Doi: https://doi.org/10.1002/hep.27422

9. El Khoury AC, Vietri J, Prajapati G. Health-related quality of life in patients with hepatitis $\mathrm{C}$ virus infection in Brazil. Rev Panam Salud Publica. 2014;35(3):200-6.

10. Souza NP, Villar LM, Garbin AJ, et al. Assessment of healthrelated quality of life and related factors in patients with chronic liver disease. Braz J Infect Dis. 2015;19(6):590-595. Doi: https://doi.org/10.1016/j.bjid.2015.08.003

11. DiBonaventura MD, Wagner JS, Yuan Y, et al. Humanistic and economic impacts of hepatitis $\mathrm{C}$ infection in the United States. J Med Econ. 2010;13(4):709-718. Doi: https://doi. org/10.3111/13696998.2010.535576

12. Foster GR, Goldin RD, Thomas HC. Chronic hepatitis C virus infection causes a significant reduction in quality of life in the absence of cirrhosis. Hepatology. 1998;27(1):209-12. Doi: https://doi.org/10.1002/hep.510270132

13. Alonso J, Ferrer M, Gandek B, et al. Health-related quality of life associated with chronic conditions in eight countries: results from the International Quality of Life Assessment 
(IQOLA) Project. Qual Life Res. 2004;13(2):283-298. Doi: https://doi.org/10.1023/B:QURE.0000018472.46236.05

14. Ware J KM, Turner-Bowker D, Gandek B. SF-12v2: how to score version 2 of the SF-12 health survey. Quality Metric Incorporated Health Assessment Lab. 2002.

15. Mhatre SK, Sansgiry SS. Development of a conceptual model of health-related quality of life among hepatitis $C$ patients: A systematic review of qualitative studies. Hepatol Res. 2016;46(1):29-39.

16. Sgorbini M, O’Brien L, Jackson D. Living with hepatitis $\mathrm{C}$ and treatment: the personal experiences of patients. J Clin Nurs 2009;18(16):2282-91. Doi: https://doi. org/10.1111/j.1365-2702.2009.02806.x

17. Sheppard K, Hubbert A. The patient experience of treatment for hepatitis C. Gastroenterol Nurs. 2006;29(4):309-15. Doi: https://doi.org/10.1097/00001610-200607000-00008

18. Dudley T, Chaplin D, Clifford C, et al. Quality of life after liver transplantation for hepatitis $\mathrm{C}$ infection. Qual Life Res. 2007;16(8):1299-308. Doi: https://doi.org/10.1007/ s11136-007-9244-y

19. Blacklaws $H$, Veysey $H$, Skinner V, et al. Interferon treatment for chronic hepatitis C: a family impact study. Gastroenterol
Nurs 2009;32(6):377-383. Doi: https://doi.org/10.1097/ SGA.0b013e3181c10759

20. Kinder M. The lived experience of treatment for hepatitis C. Gastroenterol Nurs. 2009;32(6):401-8. Doi: https://doi. org/10.1097/SGA.0b013e3181c1497f

21. Blasiole JA, Shinkunas L, Labrecque DR, et al. Mental and physical symptoms associated with lower social support for patients with hepatitis C. World J Gastroenterol. 2006;12(29):4665-72. Doi: https://doi.org/10.3748/wjg. v12.i27.4665

22. Bourliere M, Bronowicki JP, de Ledinghen V, et al. Ledipasvir-sofosbuvir with or without ribavirin to treat patients with HCV genotype 1 infection and cirrhosis nonresponsive to previous protease-inhibitor therapy: a randomised, double-blind, phase 2 trial (SIRIUS). Lancet Infect Dis 2015;15(4):397-404. Doi: https://doi.org/10.1016/ S1473-3099(15)70050-2

23. Younossi ZM, Stepanova M, Nader F, et al. Patient-reported outcomes in chronic hepatitis C patients with cirrhosis treated with sofosbuvir-containing regimens. Hepatology. 2014;59(6):2161-9. Doi: https://doi.org/10.1002/hep.27161 